konstanten von $K=0,88$ entspricht, sollte man mit Hilfe der Konzentrationsabhängigkeit des NMR-Spektrums in dem Bereich von $C_{\mathrm{MePy}}=0,88$ (Mole $/ l$ ) einen „Umschlag“ des Spektrums feststellen können. Dieser Umschlag wird tatsächlich beobachtet (Abb. 2) : es findet bei zwei Signalpaaren der aromatischen Protonen (um $8 \mathrm{ppm}, \mathrm{CDCl}_{3}$, TMS als innerer Standard) eine Intensitätsumkehr statt. Das Spektrum des Übergangs weist zusätzliche Signalaufspaltungen auf. Ein analoger Umschlag konnte bei verschieden konzentrierten Pyrenlösungen nicht beobachtet werden. Wahrscheinlich ist eine derartige Veränderung des Pyren-NMR-Spektrums aus Gründen der höheren Symmetrie der Anordnung der Wasserstoffatome gar nicht möglich.

Aus Symmetriegründen ist wahrscheinlich nur bei diskreten Konfigurationen der Pyrenmoleküle zueinander eine Absorption des dimeren Pyrens erlaubt ${ }^{8}$. Einen Hinweis für die Richtigkeit dieser Vorstellung

8 T. Azumi u. H. Auzmi, Bull. chem. Soc. Japan 39, 1829 [1966].

9 M. Wilk u. J. Rochlitz, Liebigs Ann. Chem. 701, 6 [1967].

10 J. Ferguson, J. chem. Physics 44, 2677 [1966]. gibt das Absorptionsspektrum von kristallinem Pyren, das nur ca. $5 \mathrm{~nm}$ längerwellig liegt als das Lösungsspektrum. Weiterhin ist die geringe Lösungsmittelabhängigkeit der Absorptionsspektren dieser unpolaren Aromaten bekannt ${ }^{9}$, so daß von einer Wechselwirkung von ca. $7 \mathrm{kcal} / \mathrm{Mol}$ nur minimale Veränderungen des Absorptionsspektrums zu erwarten wären.

Es ist also nach den vorliegenden Ergebnissen anzunehmen, daß - ebenso wie Pyren und 3-Methylpyren - eine Reihe von Excimeren bildenden Kohlenwasserstoffen im Grundzustand assoziiert ist ${ }^{10}$. Das Assoziationsgleichgewicht wird im angeregten Zustand in Richtung einer stärkeren Assoziation verschoben ganz ähnlich wie bei anderen Änderungen von Gleichgewichtskonstanten durch elektronische Anregung ${ }^{11}$.

Herrn Dr. WILK ist für kritische Diskussionsbeiträge und die Ermöglichung dieser Arbeit, Frau KöHLER für die Aufnahme der NMR-Spektren zu danken.

11 Tн. Förster, Z. Elektrochem. angew. physik. Chem. 54, 42, 531 [1950] ; A. Weller, Z. Elektrochem., Ber. Bunsenges. physik. Chem. 61, 956 [1957]; N. Mataga u. S. Tsuno, Bull.chem. Soc. Japan 30, 368 [1957] ; M. Wilk u. J. RochLITz, Liebigs Ann. Chem. 699, 107 [1966].

\section{Flavon-C-Glykoside in den Blättern von Vitis cinerea Darwin *}

\author{
H. W \\ Institut für Pharmazeutische Arzneimittellehre \\ der Universität München \\ und F. YAP und A. REICHARDT \\ Institut für Züchtungsforschung der Bay. Landesanstalt \\ für Wein-, Obst- und Gartenbau, Würzburg \\ (Z. Naturforschg. 22 b, 988-989 [1967] ; eingegangen am 6. Juni 1967)
}

Im Rahmen von Untersuchungen über die biochemischen Grundlagen der Reblausresistenz bei Reben haben $\mathrm{Y}_{\mathrm{AP}}$ und REICHARDT ${ }^{1}$ das Blattmaterial von über 70 artreinen Vitis-Sorten und ihren Bastarden qualitativ auf Flavonoide und Phenolcarbonsäuren untersucht. Einige Verbindungen wurden chromatographisch, durch hydrolytischen Abbau und spektrophotometrisch näher charakterisiert. Hiernach war das Vorliegen von Isoquercitrin und weiteren Quercetin- bzw. Kämpferolglykosiden wahrscheinlich. Dabei fielen vor allem die Reblaus-resistenten Vitis-Arten Vitis cinerea und Vitis berlandieri durch eine abweichende Flavonführung auf. Ähnliche chromatographische Untersuchungen über die Flavonverteilung in Reblaus-resistenten Vitis-Arten wurden von Henke ${ }^{2}$ durchgeführt.

Bei einer erneuten Untersuchung konnten in dem methanolischen Auszug der Blätter von Vitis cinerea papierchromatographisch (BEW 4:1:5) fünf Flavon-

* VI. Mitteilung über Flavon-C-Glykoside.

1 F. Yap u. A. Reichardt, Der Züchter 34, 143 [1964].

2 O. Henke, Phytopathol. Z. 32, 149 [1958] ; 41, 253 [1959]. verbindungen nachgewiesen werden. Die Isolierung aller Verbindungen gelang durch Chromatographie an Cellulosesäulen, die Identifizierung durch Schmelzpunkt, Elementaranalyse, UV-, IR- und NMR-Spektroskopie sowie Vergleich mit authentischen Verbindungen. Davon gehören vier Flavonoide der bisher nur sporadisch im Pflanzenreich aufgefundenen C-Glykosid-Reihe ${ }^{3}$ an.

Die isolierten Verbindungen sind folgende:

1. Vitexin (8-C- $\beta$-D-Glucopyranosyl-5.7.4'-Trihydroxyflavon).

Schmp. $264-265^{\circ} \mathrm{C}, \mathrm{C}_{21} \mathrm{H}_{20} \mathrm{O}_{10}(432,37)$.

Ber. C 58,33 H 4,66; Gef. C 58,08 H 4,80.

UV-Spektrum in Meth. (Max. $\lambda=270,334 \mathrm{~m} \mu$, $\log \varepsilon=4,20,4,20)$.

2. Iso-Vitexin (Saponaretin) (6-C- $\beta$-D-Glucopyranosyl5.7.4'-Trihydroxyflavon).

Schmp. $=235^{\circ}, \mathrm{C}_{21} \mathrm{H}_{20} \mathrm{H}_{10} \cdot \mathrm{H}_{2} \mathrm{O}(450,38)$.

Ber. C 56,00 H 4,92; Gef. C 55,83 H 4,93.

UV-Spektrum: (Max. $\lambda=272,337 \mathrm{~m} \mu, \log \varepsilon=$ $4,12,4,15)$.

3. Orientin (Lutexin) (8-C- $\beta$-D-Glucopyranosyl$5.7 .3^{\prime} \cdot 4^{\prime}$-Tetrahydroxyflavon).

Schmp. $=263-265^{\circ}$ (Zers.), $\mathrm{C}_{21} \mathrm{H}_{20} \mathrm{O}_{11}(448,37)$. Ber. C 56,25 H 4,50; Gef. C 56,10 H 4,88.

UV-Spektrum: (Max. $\lambda=258,270,350 \mathrm{~m} \mu$, $\log \varepsilon=4,18,4,23,4,26)$.

4. Iso-Orientin (Homo-Orientin, Lutonaretin) (6-C- $\beta$-DGlucopyranosyl-5.7.3'.4'-Tetrahydroxyflavon).

${ }^{3}$ H. W AGNER, in : Comparative Biochemistry Flavonoid-C-Glycosides, ed. T. Swain, Pergamon Press, Oxford 1966, S. 309. 
Schmp. $=235^{\circ}, \mathrm{C}_{21} \mathrm{H}_{20} \mathrm{O}_{11} \cdot \mathrm{H}_{2} \mathrm{O}(466,38)$.

Ber. C 54,06 H 4,75; Gef. C 54,25 H 4,36.

UV-Spektrum: (Max. $\lambda=259,272,350 \mathrm{~m} \mu$, $\log \varepsilon=4,23,4,32,4,35)$.

5. Iso-Quercitrin (3.5.7.3'.4'-Pentahydroxy-3-mono$\beta$-D-glucopyranosid) .

Schmp. $=221-222^{\circ}, \mathrm{C}_{21} \mathrm{H}_{20} \mathrm{O}_{12}(464,37)$.

Ber. C 54,31 H 4,34; Gef. C 54,05 H 4,59.
UV-Spektrum: $($ Max. $\lambda=258,360 \mathrm{~m} \mu, \log \varepsilon=$ $4,35,4,32)$.

Über Einzelheiten dieser Untersuchungen, die fortgesetzt werden, berichten wir an anderer Stelle.

Der Deutschen Forschungsgemeinschaft und dem Fonds der Chemischen Industrie danken wir für die Förderung dieser Arbeit.

\section{Zur Biosynthese von Phosphonoaminosäuren. Die Verteilung der Radioaktivität in Amino- äthylphosphonsäure nach Einbau von positions- markierter Glucose durch Tetrahymena}

\author{
A. Trebst und F. Geike
}

Pflanzenphysiologisches Institut der Universität Göttingen, Abteilung Biochemie der Pflanzen

(Z. Naturforschg. 22 b, 989—991 [1967] ; eingegangen am 3. Juni 1967)

In den letzten Jahren sind Phosphonoaminsäuren aus den verschiedensten Organismen isoliert worden ${ }^{1-9}$. Charakteristikum dieser Substanzklasse ist die stabile $\mathrm{P}$ - C-Bindung, die im Gegensatz zu allen anderen organischen Phosphorverbindungen der Zelle selbst durch 72 -stdg. Kochen in 6- $n$. $\mathrm{HCl}$ nicht zu spalten ist. Formal kann man 2-Aminoäthylphosphonsäure (AEP) sowohl als Analogon von Phosphoäthanolamin, als auch von $\beta$-Alanin auffassen.

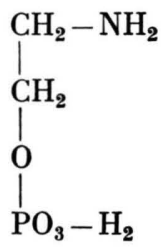<smiles>NCCC(=O)O</smiles><smiles>NCCC(=O)O</smiles>

\section{Phospho-} äthanolamin

Aminoäthylphosphonsäure $\beta$-Alanin

Das Vorkommen in Lipiden ${ }^{1-8}$ läßt die Analogie zu Phosphoäthanolamin wahrscheinlicher erscheinen, obwohl AEP auch in Proteinfraktionen ${ }^{3,9}$ gefunden wurde, aus denen sie jedoch durch Behandlung mit Pronase und Trypsin nicht freizusetzen war ${ }^{3}$.

Kinetische Untersuchungen mit ${ }^{32} \mathrm{P}$-markiertem Phosphat an Tetrahymena zeigten einen schnelleren Einbau in lipidgebundene, als in freie AEP, woraus geschlossen wurde, daß die Biosynthese von AEP an Lipidvor-

1 M. Horiguchi and M. Kandatsu, Nature [London] 184, 901 [1959].

2 M. Kandatsu and M. Horiguchi, Agric. biol. Chem. [Tokyo] 26, 721 [1962].

3 H. Rosenberg, Nature [London] 203, 299 [1964].

4 J. S. Kittredge and R. R. Hughes, Biochemistry 3, 991 [1964].

5 J. S. Kittredge, E. Roberts, and D. G. Simonsen, Biochemistry 1, 624 [1962]. läufern erfolgt ${ }^{3}$. Auf Grund der bisher veröffentlichten Daten sowie des Verhaltens von Modellverbindungen und Phospholipiden, hat SEgal ${ }^{10}$ einen Biosynthese-Mechanismus über ein Rearrangement von Phosphatidyläthanolamin zu Phosphatidyl-AEP vorgeschlagen. Über die Herkunft des C-Gerüsts liegen bisher jedoch noch keine experimentellen Informationen vor.

Es soll hier über erste Versuche zur Biosynthese von AEP in Tetrahymena berichtet werden, in denen positionsmarkierte Glucose an wachsende Kulturen von Tetrahymena pyriformis Wh 14 verfüttert, die gebildete AEP isoliert und zur Bestimmung der ${ }^{14} \mathrm{C}$-Verteilung chemisch abgebaut wurde. Dazu wurde Tetrahymena in $1-l-$ E rle nmeyer-Kolben in $300 \mathrm{ml}$ Medium mit $6 \mathrm{~g}$ Proteose-Pepton, 1,5 g Hefeextrakt und $0,6 \mathrm{~g}$ Glucose angezogen. Nach $36 \mathrm{Stdn}$. Inkubation bei $37^{\circ} \mathrm{C}$ wurde abzentrifugiert und weitere $10 \mathrm{Stdn}$. in neuem Medium mit $2 \mathrm{~g}$ Proteose-Pepton, $0,3 \mathrm{~g}$ Hefeextrakt und $0,1 \mathrm{~g}$ Glucose mit $0,03 \mathrm{mC}{ }^{14} \mathrm{C}$ inkubiert. Die Zellen wurden dann bei $2000 \mathrm{~g}$ abzentrifugiert und mit $250 \mathrm{ml}$ 6-n. HCl 24. Stdn. am Rückfluß gekocht. Das eingeengte Hydrolysat wurde in 1-n. $\mathrm{HCOOH}$ auf eine Dowex-50-H-Säule $(1 \cdot 25 \mathrm{~cm})$ gegeben und die beiden bisher in Tetrahymena beschriebenen Phosphonoaminosäuren, AEP und 2-Amino-3-Phosphonopropionsäure $(\mathrm{APP})^{4}$, zunächst mit Wasser, dann mit $0,6-n$. $\mathrm{HCl}$ von der Säule eluiert. Die AEP-enthaltenden Fraktionen wurden konzentriert, auf eine Dowex-1-Ac-Säule $(1 \cdot 32 \mathrm{~cm})$ gegeben und mit $0,001-n$. Essigsäure eluiert. Die weitere Reinigung der AEP erfolgte nach Zugabe von Träger-AEP durch Umkristallisation aus 60-proz. Äthanol bis zur konstanten spezifischen Aktivität. Dieser Aufarbeitungsgang - eine Kombination und Modifikation bekannter Methoden ${ }^{1,4}$ - wurde in Ansätzen mit ${ }^{32} \mathrm{P}$-markiertem Phosphat ausgearbeitet. Die Reinigungsschritte sind in Tab. 1 aufgeführt.

Danach wurden unter den angewandten Bedingungen von Tetrahymena zwischen 3 und $6 \%$ des aufgenommenen Phosphats in 2-AEP und $0,3 \%$ in APP eingebaut. Nach Kandatsu und Horiguchi ${ }^{2}$ kann Tetra-

6 G. Rouser, G. Kritchevsky, D. Heller, and E. Lieber, J. Amer. Oil Chemists' Soc. 40, 425 [1963].

7 T. Hori, O. Itasaka, H. Inoue, and K. Yamada, J. Biochemistry [Tokyo] 56, 477 [1964].

8 A. J. De Koning, Nature [London] 210, 113 [1966].

9 L. D. Quin, Science [Washington] 144, 1133 [1964].

10 W. Segal, Nature [London] 208, 1284 [1965]. 